\title{
EDITORIAL
}

\section{Can determination of gene polymorphism be of practical value in tailoring the treatment of HSCT patients?}

\author{
Bone Marrow Transplantation (2015) 50, 161-162; doi:10.1038/ \\ bmt.2014.295
}

Matching the donor and recipient in HLA alleles lowers the risk of HSCT to the level of sibling transplantation. Nonetheless, some patients develop GVHD and suffer from prolonged immune system reconstitution, which causes fatalities. The discovery of the effect of gene polymorphism on the gene-transcribing potential or structure of encoded proteins led to the hypothesis that, in addition to the presence of disparities in HLA specificities, the genetically controlled magnitude and pace of the immune response do play a role.

In spite of more than 15 years of research in the field of gene polymorphism, there is no recommendation for the clinical implementation of the studies. This may be due to the fact that not only one gene's characteristics but those of a number of genes working along the same signaling pathway should be considered, which makes the single-gene polymorphism analysis approach unfeasible. Alternatively it can be assumed that the effect of gene polymorphism-associated features may be seen only in patients with some well-defined clinical situations. Indeed, 13 CA microsatellite polymorphism at position +875 within the first intron of the IFNY gene of the patients influences the risk of CMV reactivation, but this effect is only clinically relevant if the polymorphic feature, representing a low level of IFNy production, ${ }^{1}$ goes together with low CD4+ lymphocyte count. ${ }^{2}$ On studying the NOD2/CARD15 gene polymorphism we learned that the presence of SNP 13 (3020insC, Leu1007 fsins C) mutation in patients as well as donors constitutes a risk factor of septic complications in HSCT patients exposed to deadly bacteria. ${ }^{3}$ NOD2/CARD15, if triggered by muramyl dipeptide, activates two pathways: one going to mitochondrial antiviral-signaling protein/ Interferon regulatory factors (MAVS/IFR) and the other to nuclear factor $\mathrm{KB}$ and activating protein-1; the latter ones are the transcription factors of several molecules involved in the immune response including IFN ${ }^{4}{ }^{4}$ The natural immune (IFN $\beta$ ) response makes the first line of resistance and IFNy generation alert adaptive immunity. Signal transduction within the cells aimed at activation of different DNA-binding molecules (transcription factors, STATs) uses different pathways employing kinases belonging to several families. Clinical observation of SNPs in the NOD2/CARD15 gene suggests that single-nucleotide polymorphism (SNP) 13 impairs the natural immunity mechanism, but not the one leading to cytokine production. ${ }^{5}$ This observation helps in understanding the mechanism of frequently fatal septic complications seen in patients with SNP 13. The results of the abovementioned observations show that the effect of the above-quoted gene polymorphisms may be clinically seen only in situations associated with a higher risk of infections.

Probably the best example of the biological significance of gene diversity in man, depending on the situation they face, is provided by the paper published in this issue entitled 'Caspase-8 polymorphisms result in reduced Alemtuzumab-induced T-cell apoptosis and worse survival after transplantation'. ${ }^{6}$

Apoptosis inevitably regulates the life span of cells. The apoptotic signal is processed within the cells, leading to the sequential activation of caspases executing cell apoptosis.
Caspase 8 has a central position in the apoptotic pathway and six-nucleotide deletion within the promoter region of this gene affects gene transcription, consequently influencing the homeostasis of cells, including lymphocytes and macrophages. Deletion polymorphism of this gene has clinical relevance in the lower susceptibility to some cancers. ${ }^{7}$ The beauty of the abovementioned paper lies in the fact that it finds the significance of this deletion mutation in the susceptibility to immunosuppression exerted by Alemtuzumab. The mechanism of this immunosuppression depends at least in part on the induction of apoptosis. Quite understandably, donors with six-nucleotide deletion within the Caspase 8 promoter ( $r 3834129,-652$ del AGTAAG) provide the recipients with $T$ lymphocytes resistant to activation-induced cell death. Therefore, Alemtuzumab has a restricted effectivity, which translates into poorer transplantation outcomes in patients who receive transplants from donors with Caspase 8 deletion mutation. The authors have documented the mechanism involved in resistance to apoptosis of lymphocytes that have the Caspase 8 mutation, showing it in in vitro experiments.

The other important insight offered by the study is that deletion polymorphism of the Caspase 8 gene can have an opposite effect on the fate of patients, depending on the clinical situation they face. It is known that the presence of deletion mutation making $T$ cells more resistant to activation-induced cell death is associated with reduced susceptibility to some cancers, ${ }^{7}$ which benefits mutation-harboring individuals. On the contrary, as shown in the paper by Shaw et al., ${ }^{6}$ the presence of the mutation in patients in need of Alemtuzumab-induced immunosuppression adversely affects the treatment outcome.

This paper points to the importance of determining the polymorphism of genes encoding proteins relevant for the disease pathomechanism and treatment. If the polymorphism affects the function of encoded proteins, the determination of the allelic diversity of individuals may help in tailoring the treatment to the needs of individual patients.

\section{CONFLICT OF INTEREST}

The authors declare no conflict of interest.

\author{
A Lange ${ }^{1,2}$ and E Jaskula ${ }^{1,2}$ \\ ${ }^{1}$ L. Hirszfeld Institute of Immunology and Experimental Therapy, \\ Polish Academy of Sciences, Wroclaw, Poland and \\ ${ }^{2}$ Lower Silesian Center for Cellular Transplantation with National \\ Bone Marrow Donor Registry, Wroclaw, Poland \\ E-mail: lange@iitd.pan.wroc.pl
}

\section{REFERENCES}

1 Pravica V, Asderakis A, Perrey C, Hajeer A, Sinnott PJ, Hutchinson IV. In vitro production of IFN- $\gamma$ correlates with CA repeat polymorphism in the human IFN- $\gamma$ gene. Eur J Immunogenet 1999; 26: 1-3.

2 Jaskula $E$, Dlubek D, Duda D, Bogunia-Kubik K, Mlynarczewska A, Lange A Interferon gamma 13-CA-repeat homozygous genotype and a low proportion of $\mathrm{CD} 4(+)$ lymphocytes are independent risk factors for cytomegalovirus reactivation with a high number of copies in hematopoietic stem cell transplantation recipients. Biol Blood Marrow Transpl 2009; 15: 1296-1305.

3 Jaskula E, Lange A, Kyrcz-Krzemien S, Markiewicz M, Dzierzak-Mietla M, Jedrzejczak WW et al. NOD2/CARD15 single nucleotide polymorphism 13 (3020insC) is associated with risk of sepsis and single nucleotide polymorphism 8 
(2104C $>$ T) with herpes viruses reactivation in patients after allogeneic hemato poietic stem cell transplantation. Biol Blood Marrow Transpl 2014; 20: 409-414.

4 Correa RG, Milutinovic S, Reed JC. Roles of NOD1 (NLRC1) and NOD2 (NLRC2) in innate immunity and inflammatory diseases. Biosci Rep 2012; 32: 597-608.

5 Mayor NP, Shaw BE, Madrigal JA, Marsh SGE. NOD2 polymorphisms and their impact on haematopoietic stem cell transplant outcome. Bone Marrow Res 2012 2012: 180391
6 Shaw BE, Lee F, Krishnamurthy S, Byrne JL, Seedhouse C, Mayor NP et al. Caspase-8 polymorphisms result in reduced Alemtuzumab-induced T-cell apoptosis and worse survival after transplantation. Bone Marrow Transpl 2014, 1-7.

7 Zhang F, Yang Y, Guo C, Wang Y. CASP8 $-6526 \mathrm{~N}$ del polymorphism and cancer risk: a meta-analysis of 30 case-control studies in 50,112 subjects. Mutagenesis 2012; 27 559-566. 